\title{
The effects of background visual roll stimulation on postural and manual control and self-motion perception
}

\author{
FRED H. PREVIC \\ Armstrong Laboratory, Brooks Air Force Base, Texas \\ ROBERT V. KENYON and ERWIN R. BOER \\ University of Illinois, Chicago, Illinois \\ and \\ BEVERLY H. JOHNSON \\ KRUG International, San Antonio, Texas
}

\begin{abstract}
The effects of background visual roll stimulation on postural control, manual control, and selfmotion perception were investigated in this study. In the main experiment, 8 subjects were exposed to wide field-of-view background scenes that were tilted and static, continuously rotating, or sinusoidally rotating at frequencies between 0.03 and $0.50 \mathrm{~Hz}$, as well as a baseline condition. The subjects performed either a postural control task (maintain an upright stance) or a manual control task (keep an unstable central display horizontally level). Root-mean square (RMS) error in both the postural and manual control tasks was low in the static tilt condition and extremely high in response to continuous rotation. Although the phases of the postural and manual responses were highly similar, the power and RMS error generated by the sinusoidal visual background stimulation peaked at a lower frequency in the postural task. Vection ratings recorded at the end of the postural and manual trials somewhat paralleled the frequency tuning differences between tasks, which a subsequent experiment showed to be the result of the differential motion of the central display rather than the differential positioning of the subject. In general, these results show that the dynamic characteristics of visual orientation systems vary according to the specific motor and/or perceptual system investigated.
\end{abstract}

The effects of visual information on spatial orientation in humans have been widely studied (see Dichgans \& Brandt, 1978, for a review). In terms of their dynamic range, visually mediated perceptual-motor responses are generally characterized by their lowpass nature; beyond $0.25 \mathrm{~Hz}$, for example, these responses are typically associated with large phase lags and low gains (Berthoz, Pavard, \& Young, 1975; Cheung \& Howard, 1991; Dichgans \& Brandt, 1978; Dichgans, Mauritz, Allum, \& Brandt, 1976; Huang \& Young, 1988; Lestienne, Soechting, \& Berthoz, 1977; St. Cyr \& Fender, 1969; Van Asten, Gielen, \& Denier Van Der Gon, 1988; Xerri, Borel, Barthelemy, \& Lacour, 1988; Zacharias \& Young, 1981). By comparison, the compensatory oculomotor response

We thank the following individuals for their contributions to this study: Kent Gillingham and Lisa Weinstein (manuscript review); Karen Lesiter (typing); Joe Fischer and Carolyn Oakley (statistical consulting); and Stan Singleton and Steve Stranges (engineering support). This research was supported by intramural Grant 88-LAB-03 from the Air Force Office of Scientific Research. Portions of these data were presented at the May 1991 62nd Annual Scientific Meeting of the Aerospace Medical Association in Cincinnati, $\mathrm{OH}$. Address correspondence to F. H. Previc, Crew Technology Division, Armstrong Laboratory, Brooks AFB, TX 78235-5000. to vestibular stimulation exhibits a near-zero phase lag and near-unity gain at $1 \mathrm{~Hz}$ (see Howard, 1986).

Although visual orientation responses may be slower than their vestibularly mediated counterparts, they can exhibit considerable variability in their dynamic properties, depending on the particular visuomotor system in question. For example, the response to visual roll stimulation need not be as dominated by low-frequency inputs as the corresponding response to yaw motion, given that the otolithic response to motion about the visual axis is veridical in the upright position (see Raphan \& Cohen, 1985). But even when only roll motion is considered, the perception of self-rotation (vection) begins long after the initial postural reaction (Clement, Jacquin, \& Berthoz, 1985; Previc \& Mullen, 1991) and is even more delayed relative to the corresponding oculomotor reflex (ocular torsion), whose lag approaches zero at $0.2 \mathrm{~Hz}$ (Cheung \& Howard, 1991).

Despite the longer latency of vection relative to postural change, these two "whole-body" responses appear to be similar to each other in that (1) both are greater in response to roll and pitch than to fore-aft linear stimulation (Previc, 1992); (2) both are influenced most by distant, peripheral visual motion (Delorme \& Martin, 1986); 
and (3) the amplitudes of both responses increase linearly as a function of increasing visual background velocity up to $\sim 20$ dps (degrees per second) (Clement et al., 1985). By contrast, previous research has shown that background visual effects on manual control differ from vection in a number of respects, including the larger impact of central stimulation on manual control (Donnelly \& Previc, 1992; Kenyon \& Kneller, 1993) and the greater gravitational effect (i.e., shifting from a seated to a supine position) on vection (Kenyon, unpublished data). Visually mediated manual control effects also differ from their postural counterparts in that they appear to be much less strongly mediated by the vestibulospinal reflex pathways (compare Weinstein, Previc, Simpson, Lyons, \& Gillingham, 1991 with Thoden, Dichgans, \& Savidis, 1977).

On the basis of the above relationships, the temporal frequency tuning of visually mediated postural changes and vection would be expected to resemble each other more closely than would the tuning of manual control responses. The natural frequency spectrum of the postural system is lowpass, with little power beyond $0.2 \mathrm{~Hz}$ (Van Asten et al., 1988), and visually generated postural sway also has been shown to peak at frequencies at or below $0.1 \mathrm{~Hz}$ (Dichgans et al., 1976; Van Asten et al., 1988). Using fore-aft visual stimulation, Berthoz et al. (1975) demonstrated that vection also exhibits lowpass temporal tuning, but in a more recent experiment that measured the dynamics of the rod-and-frame illusion when the background frame oscillated in roll (Babler \& Ebenholtz, 1989), vection-sensitive subjects reported that their illusory self-motion peaked at the highest frequency tested $(0.21 \mathrm{~Hz})$. Only a few studies have assessed visual orientation temporal tuning using the manual control system, which, because of the smaller mass of its muscle and bone tissue and its greater reliance on the monosynaptic pyramidal motor pathways, might be capable of a higher dynamic range. Babler and Ebenholtz reported an inconsistent decline in the amplitude (gain) of their subjects' manually registered frame tilt with increasing frame frequency from 0.01 to $0.21 \mathrm{~Hz}$. However, the phase lag of the manually registered perceived tilt of the rod showed a steep increase beyond $0.1 \mathrm{~Hz}$ and was comparable to previous postural phase lag estimates, except for those subjects who were highly vection-sensitive. Huang and Young (1988), meanwhile, observed predominantly lowpass visual effects in their compensatory roll-tracking task, but it is difficult to compare their results to those of Babler and Ebenholtz and others because they only varied the frequency of their nonvisual (i.e., actual) motion disturbance.

The purpose of the present study was to compare the effects of wide field-of-view (FOV) visual backgrounds on postural control, manual control, and vection in the roll plane. In order to optimize performance, we selected postural and manual tasks that required the subject to ignore the background visual input. On the basis of previous evidence, we predicted that ambient visual effects on manual tracking would be more evident at higher fre- quencies $(>0.1 \mathrm{~Hz})$ than in the case of postural control. The outcome concerning the dynamic tuning of vection was less predictable. Although vection onset is delayed relative to visually induced postural change (Previc \& Mullen, 1991)-suggesting a longer vection time-constant and greater attenuation at high temporal frequenciesmany of Babler and Ebenholtz's (1989) subjects reported the strongest roll vection at the highest frequency tested.

\section{EXPERIMENT 1}

A wide range of static and dynamic background visual roll conditions (including five sinusoidal roll frequencies) were presented to the subjects in Experiment 1, hereafter termed the "main"' experiment. We recorded two measures that have been widely studied in visual orientation experiments-postural control and vection-as well as a measure of subjects' performance during a compensatory manual tracking task used in our laboratory in several recent experiments (Donnelly \& Previc, 1992; Kenyon \& Kneller, 1993; Weinstein et al., 1991). Our major prediction was that the effects of wide FOV visual background motion on postural control would be tuned to lower temporal frequencies than in the case of manual control.

\section{Method}

Subjects. A total of 8 subjects ranging in age from 23 to 42 years participated in this experiment. All had either corrected or uncorrected binocular visual acuities equal to $20 / 25$ or better, and showed no sign of vestibular dysfunction on the sharpened Romberg test. With the exception of 2 of the experimenters (R.V.K. and B.H.J.), all the subjects were naive as to the hypotheses underlying the study.

The voluntary, fully informed consent of the subjects used in this study was obtained, as required by Air Force Regulation 169-3.

Apparatus. The experiment was conducted in the Visual Orientation Laboratory of the United States Air Force's Armstrong Laboratory, an illustration of which is contained in a previous report (Previc \& Mullen, 1991). The laboratory includes (1) a Silicon Graphics 3130 IRIS workstation; (2) a Sony $1030 \mathrm{Q}$ color video projector; (3) a subject booth containing a Draper Cine-15 viewing screen and Fresnel lens assemblage, by means of which the image was displayed, enlarged, and collimated; (4) an Advanced Mechanical Technology, Inc. (AMTI) force-measuring system, which included a 46.4 x $50.8 \mathrm{~cm}$ OR6-5 force platform, an SGA six-channel strain-gage amplifier, and a Zenith 248 computer; and (5) a simulated aircraft seat with a Measurement Systems, Inc. force-stick (Model 446) attached to the right arm. Both the video projector and the viewing ensemble were adjustable in height, so that the center of the projected image could be set at eye level for any given subject, whether standing on the force platform or seated. The basically linear output of the force-stick was lowpass filtered at $7 \mathrm{~Hz}$.

The background visual stimulus was a full-color terrain scene, whose black and white representation is shown in Figure 1. The visual scene subtended $105^{\circ}$ vertically $\times 115^{\circ}$ horizontally (i.e., the limits of the elliptical Fresnel lens assemblage), and possessed an average luminance of $2 \mathrm{~cd} / \mathrm{m}^{2}$ as measured at the approximate center of each quadrant and the center of the overall display. The visual scene contained individual texture elements of varying size and a clearly defined horizon. The horizon and the sky-ground vertical gradient were considered to be important for investigating the effects of static scene tilt on postural and manual control.

In the postural portion of Experiment 1, a small red dot subtending $1.5^{\circ}$ in diameter was superimposed on the background scene to help 


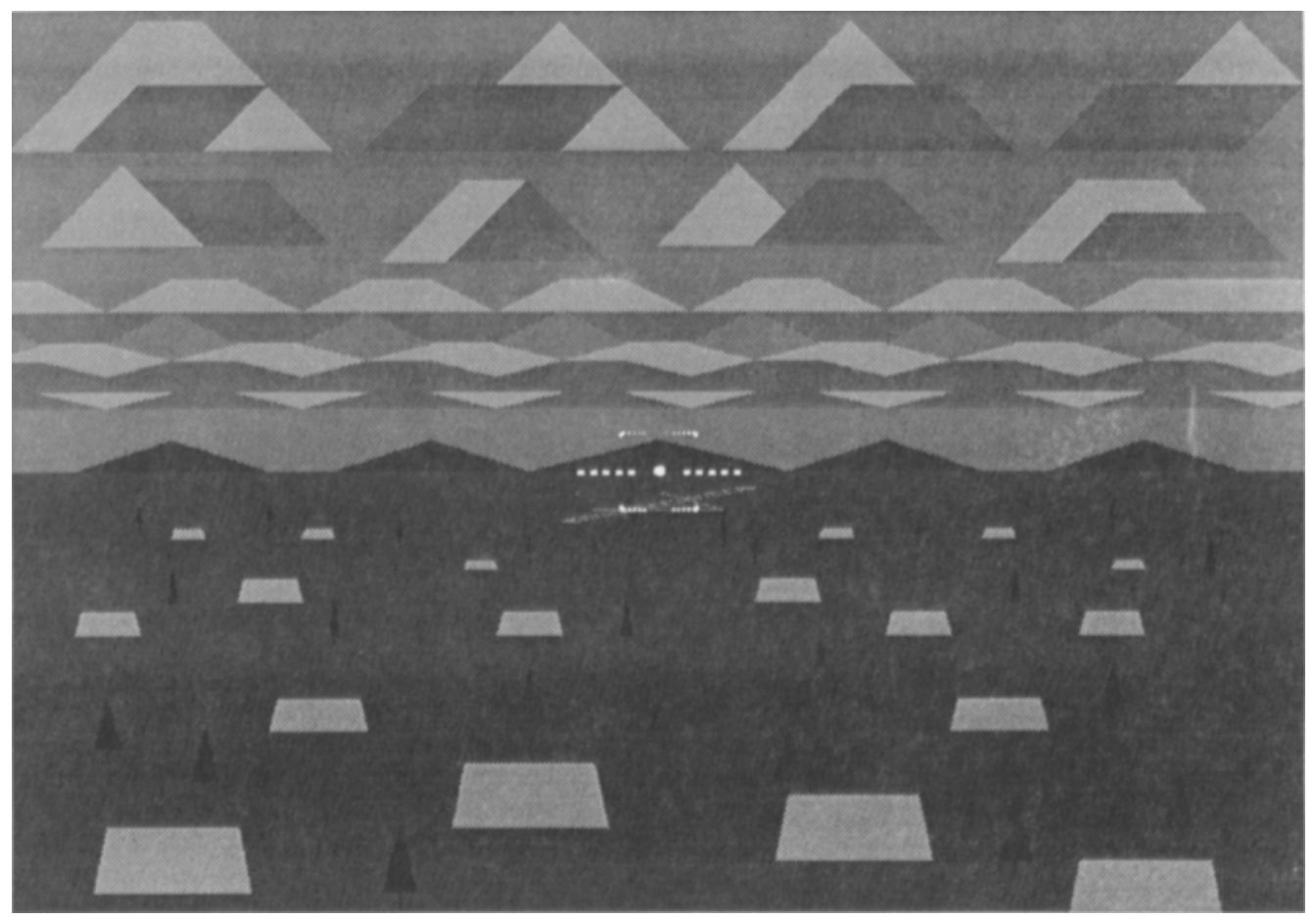

Figure 1. The visual background scene and the central display used in Experiment 1.

maintain fixation. Because of its small size and circularity, the stationary dot provided no orientational cueing. In the manual portion of Experiment 1, the fixation dot was surrounded by a red "hexagonal" display that was composed of a large horizontal dashed line and two slightly smaller parallel dashed lines that were located above and below it (see Figure 1). The outside boundary of the display subtended $10^{\circ}$ vertically $\times 22^{\circ}$ horizontally. Both the fixation dot and the larger display appeared much closer to the subject than the terrain scene because of chromostereopsis (Vos, 1960), thereby augmenting the effect of the background visual motion (Brandt, Wist, \& Dichgans, 1975; Delorme \& Martin, 1986; Heckmann \& Howard, 1991; Ohmi, Howard, \& Landolt, 1987). The central display used in the manual trials was considered to be unacceptable for the postural conditions, because (1) it would have been stationary relative to the background scene and hence could have provided additional orientational "feedback" that wasn't given by the unstable central display in the manual trials; and (2) it could have affected the perceived motion of the background scene (and, in turn, the magnitude of the postural response) by appearing to move opposite to it, as in the "induced motion" phenomenon first described by Duncker (1929).

Procedure. In both the postural and manual tasks, each subject received a total of 4 trials for each of the eight different types of visual roll stimulation. A total of 16 trials overall ( 2 trials per condition) were run during an initial 1-h session for each task, while the remaining 16 trials were run during a second session. The eight visual conditions consisted of (1) baseline, in which the background scene either moved congruently and with no roll offset relative to the central display (in the manual task) or remained stationary on the screen (in the postural task); (2) tilt, in which the scene was gradually tilted about the visual axis at a rate of $2 \mathrm{dps}$ for $10 \mathrm{sec}$ before moving congruently with the central display (in the manual task) or remaining stationary on the screen (in the postural task), albeit with a constant $20^{\circ}$ offset in both cases; (3) rotation, in which the background scene rolled continuously in one direction at $20 \mathrm{dps}$, independently of the movement of the central display; and (4-8) five sinusoidal motion conditions $(0.03,0.06,0.12,0.25$, and $0.50 \mathrm{~Hz}$ ), in which the independently moving background scene rolled sinusoidally with an amplitude of $\pm 20^{\circ}$.

Each trial was $146.5 \mathrm{sec}$ in duration, with the visual scene remaining stationary and upright during the first $10 \mathrm{sec}$. (This baseline interval was required to provide a zero reference for the postural data.) Two centrally located vertical red bars moved slowly toward each other near the end of the baseline interval and met and disappeared just as the background visual stimulus started to move. The initial rotation of the scene was in the clockwise direction on oddnumbered trials, and in the counterclockwise direction on evennumbered trials. The order of conditions was randomized across subjects, subject to the constraint that all conditions had to be presented the same number of times in each position order. Also, the presentation order for each subject was reversed for the second set of trials in each session. The two manual and two postural sessions were alternated such that the manual task was run during the first and fourth sessions for half of the subjects, and during the second and third sessions for the other half.

In the postural task, the subject was instructed to maintain an erect stance in the classic Romberg position (feet together), while centered on the force platform. The subject was then instructed to fixate the small red dot in the center of the viewing area. Each subject wore a pair of flat rubber-soled shoes that were part of a standardized set. The lateral center of pressure and the lateral postural momentwhich, assuming a constant gravitational force, basically represents the lateral translation of the center of pressure of the body on the force platform-were sampled at $15 \mathrm{~Hz}$ by means of the AMTI BEDAS-2 software package, and were referenced to their average position during the 10-sec baseline period. A total of 2,048 samples were recorded over the entire 136.5-sec trial period.

In the manual control task, the seated subject was instructed to maintain the central display at a perceived horizontal position, independent of the movement of the visual background scene. The 
subjects controlled the force-stick with the right hand, using a wholehand grip applied to the middle portion of the stick. Slight movements of the force-stick to the right or left controlled the roll position of the central display. In attempting to stabilize the central display, the subject acted as the control element in a closed-loop system that includes a first-order divergent (unstable) control plant (see Weinstein et al., 1991). Partly on the basis of training data from at least three sessions of the critical tracking task (Jex, McDonnel, \& Phatak, 1966), the lambda (instability value) of the plant was set at 2.5 , which corresponds to a moderate level of difficulty. An external forcing function that consisted of a sum-of-sinusoids pseudorandom noise disturbance was also added to the input of the manual control system. ${ }^{2}$ These two procedures were designed to (1) require constant input from the subject to keep the central display from tipping over; (2) equate the relative difficulties of the manual and postural tasks; and (3) better match the manual outputs to the broadband frequency response of the postural system. The subject was required to maintain the central display within $\pm 180^{\circ}$ of upright, or else the trial was terminated and repeated. (Such an event occurred on about $10 \%$ of the trials.) The manual response was sampled at the same rate as that used in the postural task $(15 \mathrm{~Hz})$.

A final measure that was recorded was the magnitude of the subjects' vection. The subjects rated their vection on a 5-point scale: $1=$ little or none, 2 = below average, $3=$ average, $4=$ above average, and $5=a$ great deal of vection. The ratings were obtained only during the second set of postural and manual trials of each session and were based on the complete range of vection experienced during the first set of trials. The subjects reported their vection magnitude at the completion of each trial.

\section{Results}

The effects of the various visual roll conditions on the raw postural and manual outputs are exemplified by the data shown in Figure 2. Statistical comparisons between the postural and manual responses were performed for the following output measures: (1) root-mean square (RMS) "error" relative to the mean position of the stick or postural center of pressure (a measure of response instability); (2) lateral or angular offset (bias); (3) output power at the stimulating frequency; and (4) phase offset relative to the background motion. Vection magnitude ratings in the postural and manual tasks served as the final measure that was analyzed.

\section{Manual Response}

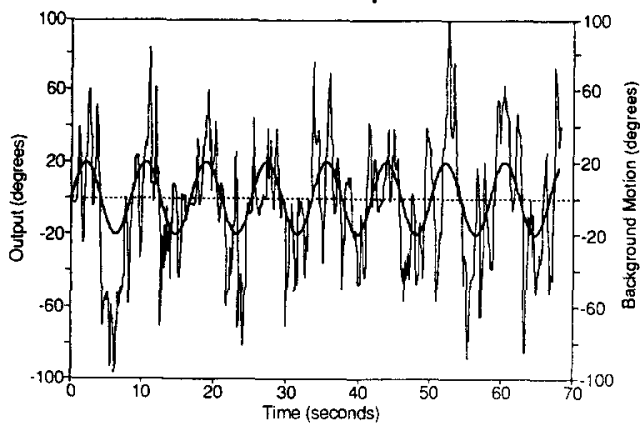

Unless otherwise specified, the above values were calculated for each of the four trials and then averaged. Repeated measures analyses of variance (ANOVAs) involving two within-subjects factors (visual condition and task) were used to test the significance of all main and interaction effects in the data.

RMS analysis. The RMS analysis was the only one that included all eight visual conditions. RMS amplitude was measured in relation to the mean center of pressure in the postural trials and the mean roll angle in the manual trials. Because the postural and manual data were expressed in units that were not directly comparable, it was necessary to transform these two sets of data into relative amplitudes. This transformation was achieved by setting each subject's highest RMS value across the eight visual conditions to 1.0 and expressing all other values as a percentage thereof. This procedure basically eliminated any absolute differences between the two measures, so that the statistical main effect of task was essentially rendered meaningless.

Figure 3 depicts the mean RMS relative amplitudes in the eight visual conditions and two tasks. The effect of the background visual scene proved very similar for the two tasks, except at the highest frequencies tested. Whereas manual RMS amplitude continued to increase up to $0.50 \mathrm{~Hz}$, postural RMS amplitude diminished beyond $0.12 \mathrm{~Hz}$. The relative postural and manual RMS values in the continuous rotation condition were comparably high ( 0.91 and 0.92 , respectively), whereas they only slightly exceeded baseline levels in the tilt condition. The relation of baseline-to-peak relative RMS amplitude was highly similar for the two tasks $(0.66$ for postural; 0.59 for manual), suggesting that the baseline task difficulties were reasonably well matched.

The RMS ANOVA yielded a highly significant main effect of visual condition $[F(7,49)=47.93, p<.001]$ and a highly significant task $\times$ visual condition interaction effect $[F(7,49)=5.49, p<.001]$. Post hoc $t$ tests showed that the mean postural and manual RMS relative

Figure 2. An illustration of the manual and postural data obtained during a portion of one trial in Experiment 1 from Subject J.A.W. in response to $0.12-\mathrm{Hz}$ background visual motion. The stimulus profile is superimposed on each of the raw data records. The postural moment values are in newton-meters. 


\section{RMS}

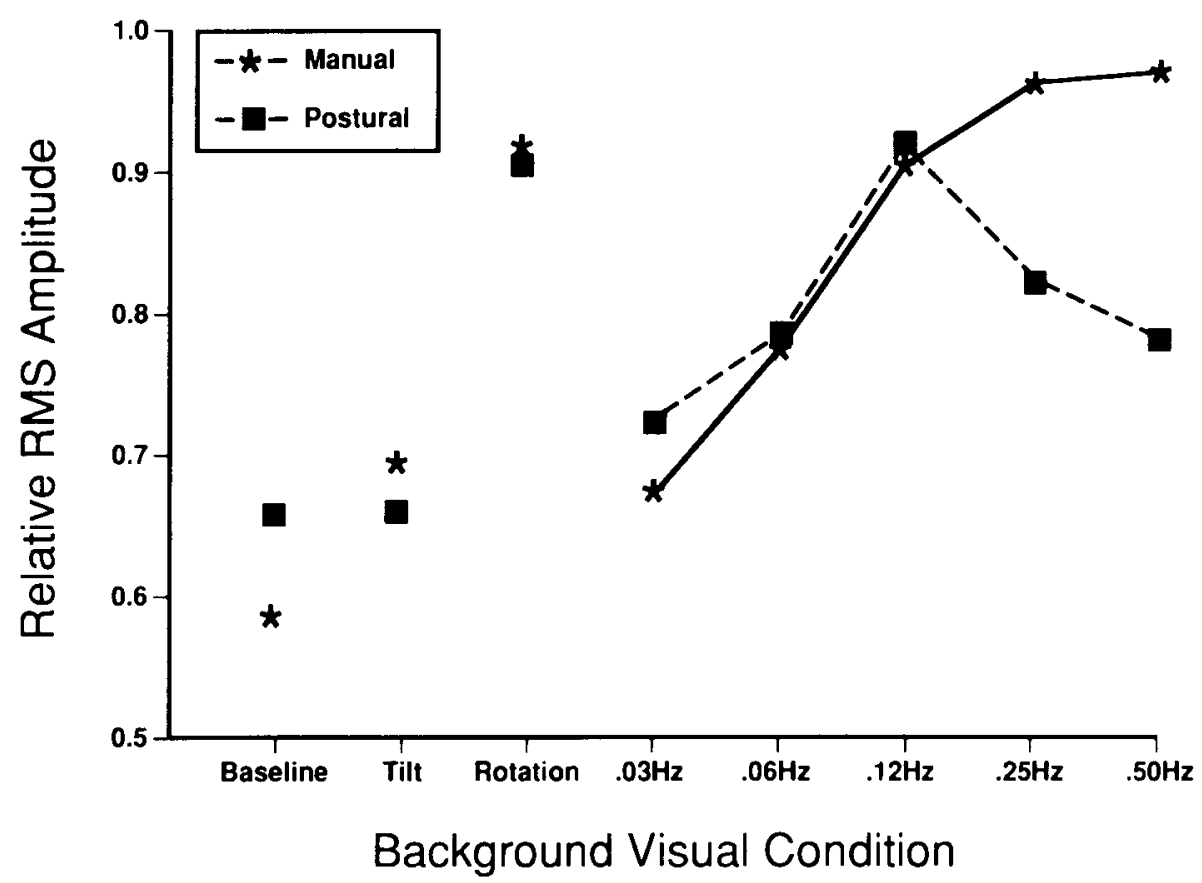

Figure 3. Postural (filled squares) and manual (*s) RMS amplitudes as a function of visual background condition. The mean relative amplitude values were calculated for each of the 8 subjects and then averaged.

amplitudes differed significantly only at $0.25 \mathrm{~Hz}(p<$ $.01)$ and $0.50 \mathrm{~Hz}(p<.001)$.

Bias analysis. The bias comparison was limited to the three nonsinusoidal visual background conditionsbaseline, tilt, and rotation. In the latter two conditions, bias was defined as the response offset in the direction of the background motion/tilt. Once again, the raw data were converted into relative values with the peak amplitude set to 1.0 .

The mean relative biases are shown in Figure 4. With few exceptions, the peak bias for both the postural and manual tasks occurred in the continuous rotation condition, whereas the tilt condition produced a large bias only in the manual task. (In actual terms, the manual biases in the rotation and tilt conditions were $14.54^{\circ}$ and $5.17^{\circ}$, respectively.) Virtually no response offsets were observed in the baseline conditions when the data were averaged across subjects.

The bias ANOVA revealed the presence of a highly significant main effect of visual condition $[F(2,14)=57.14$, $p<.001]$ and a marginally significant main effect of task $[F(1,7)=5.33, p=.05]$. Although the task $\times$ visual condition interaction did not achieve significance, the data presented in Figure 4 indicate that the difference between the two tasks was most pronounced in the tilt condition; hence, post hoc $t$ tests were performed for each visual condition. Only in the tilt condition did the difference be- tween the postural and manual relative biases attain statistical significance $(p<.05)$.

Power analysis. The magnitudes of the visually induced postural and manual responses were compared using the relative power of the responses obtained at each of the five sinusoidal frequencies. The output power of each system was used in place of the more traditional "gain" measure, because the precise input-output relationship for the postural task could not be ascertained. That is, the displacement of the subject's postural moment (center of pressure) could not be translated into the same unit (angular roll error) as the visual background input signal, because we did not additionally measure head or body (center of gravity) movement. Power was derived by means of a Fourier analysis performed at each of the five frequencies. Because the power values included the effect of the sinusoidal visual background motion as well as the contribution of the forcing function (in the manual task) and the "noise" of the human operator, the power calculated at each stimulus frequency in the baseline condition was subtracted from the power obtained in the corresponding visual condition. ${ }^{3}$ The resulting values were then transformed into relative amplitudes in the manner described previously. Since baseline power was less than $5 \%$ of the overall power in most cases, the adjusted values differed only slightly from the original ones. Moreover, the adjusted values in the manual task varied across fre- 


\section{BIAS}

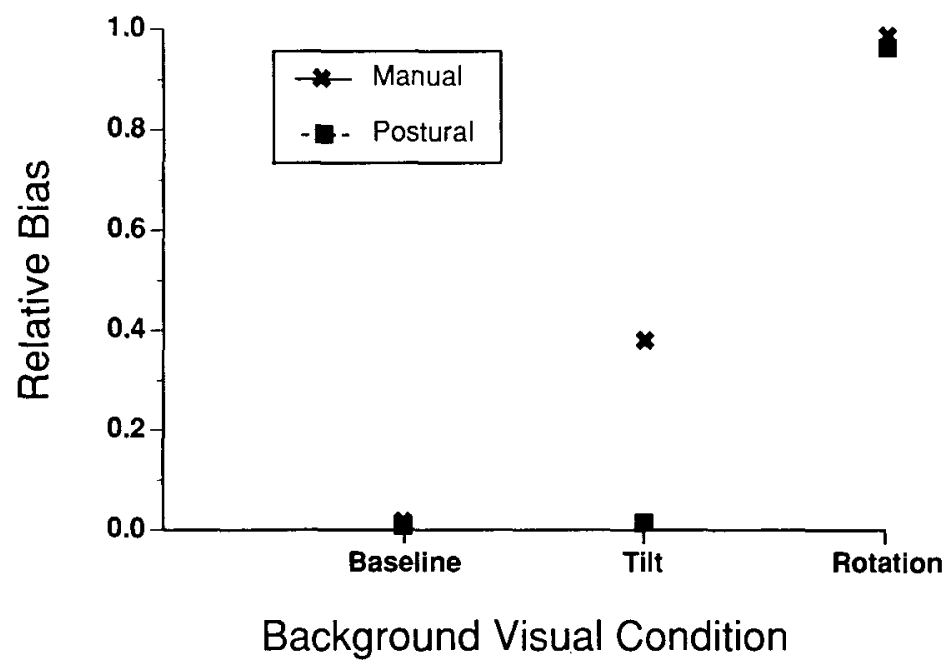

Figure 4. The relative bias of the postural and manual responses in the baseline, tilt, and rotation conditions. The mean relative bias values were calculated for each of the 8 subjects and then averaged.

quency in similar fashion to the nonadjusted (actual) gains, which generally ranged between 0.5 and 2.0 .

The mean relative power at each of the five sinusoidal visual background frequencies in the postural and manual tasks is illustrated in Figure 5. Both the postural and manual functions peaked at $0.12 \mathrm{~Hz}$, although the manual function declined more rapidly than the postural one at the lower frequencies, whereas the reverse was true at the higher frequencies.

The ANOVA performed on the relative power values revealed a highly significant effect of visual condition $[F(4,28)=29.62, p<.001]$ and a highly significant task $\times$ visual condition interaction effect $[F(4,28)=10.51$, $p<.001]$. Post hoc $t$ tests revealed that postural power significantly exceeded manual power at $0.03 \mathrm{~Hz}(p<$ $.05)$, and $0.06 \mathrm{~Hz}(p<.05)$, whereas manual power was significantly greater than postural power at $0.25 \mathrm{~Hz}(p<$ $.001)$ and $0.50 \mathrm{~Hz}(p<.01)$.

Phase analysis. By means of the same Fourier algorithm used in the power analysis, the phases of the postural and manual responses were calculated relative to the position of the background visual stimulus. Because the signal-to-noise ratio of the postural response was generally much lower than that of the manual response-and, for a few subjects at $0.50 \mathrm{~Hz}$, virtually negligible-a valid phase estimate could only be achieved by averaging all four trials together prior to the phase calculation. For comparison purposes, a similar procedure was applied to the manual data.

The mean phase results, shown in Figure 6, reveal highly similar postural and manual phase shifts as a function of stimulating frequency. All the subjects showed postural and manual phase leads at $0.03 \mathrm{~Hz}$ and a manual phase lead at $0.06 \mathrm{~Hz}$, while all but 1 subject exhibited a postural phase lead at $0.06 \mathrm{~Hz}$.

The phase ANOVA revealed a highly significant main effect of visual condition $[F(4,28)=235.84, p<.001]$. Since the task $\times$ visual condition interaction effect proved nonsignificant, no individual post hoc comparisons were performed.

Vection analysis. Vection magnitude ratings were obtained in the five sinusoidal conditions and in the continuous rotation condition (the only ones in which a systematic motion stimulus was present). The mean vection rating scores in these six visual conditions during the postural and manual tasks are shown in Figure 7. Vection ratings in the two tasks somewhat paralleled the results obtained for the RMS and power measures. Although strong vection in the rotation condition was experienced in both tasks, maximal vection occurred at a higher frequency in the manual task $(0.50 \mathrm{~Hz})$ than in the postural task $(0.25 \mathrm{~Hz})$.

The results of the vection ANOVA confirmed the existence of both a highly significant main effect of visual condition $[F(5,35)=36.47, p<.001]$ and a significant task $\times$ visual condition interaction effect $[F(5,35)=$ $2.74, p<.05]$. Post hoc $t$ tests demonstrated that the postural and manual vection ratings differed significantly only at $0.50 \mathrm{~Hz}(p<.01)$, where vection reached its peak in the manual task but began to diminish in the postural task.

\section{Discussion}

The major finding of Experiment 1 was the greater influence of mid-to-high visual background roll frequencies $(0.10-0.50 \mathrm{~Hz})$ on manual versus postural control, in spite 


\section{POWER}

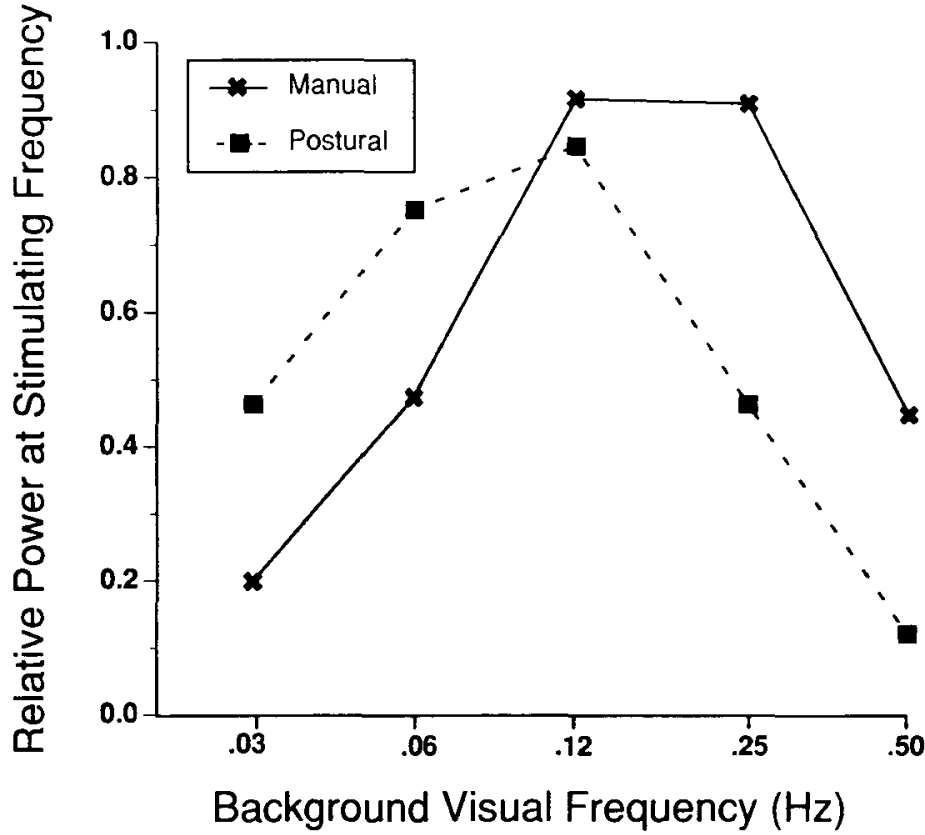

Figure 5. The mean relative power of the postural and manual responses in the five sinusoidal visual background conditions. Relative power was determined for each subject after calculating the power in response to the visual stimulus minus the power at the corresponding frequency in the baseline condition. The data were then averaged across all 8 subjects.

\section{PHASE}

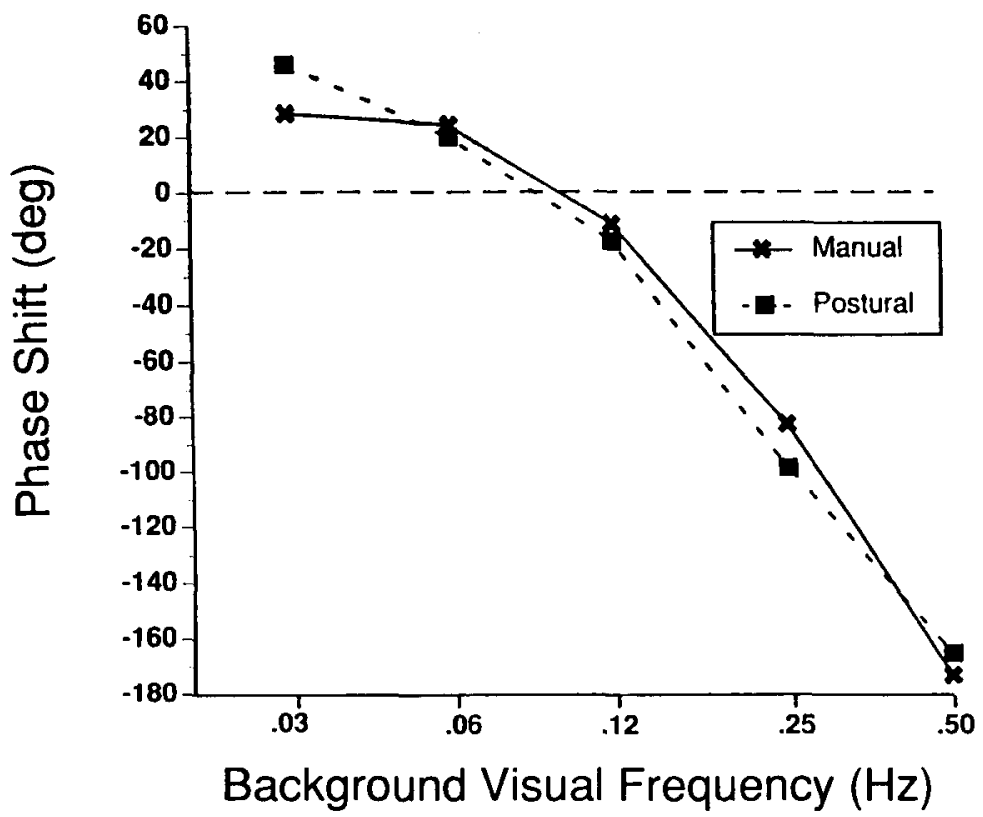

Figure 6. The mean phase of the postural and manual responses relative to the position of the visual background stimulus in the five sinusoidal visual background conditions. The data were averaged across all 8 subjects. 


\section{VECTION}

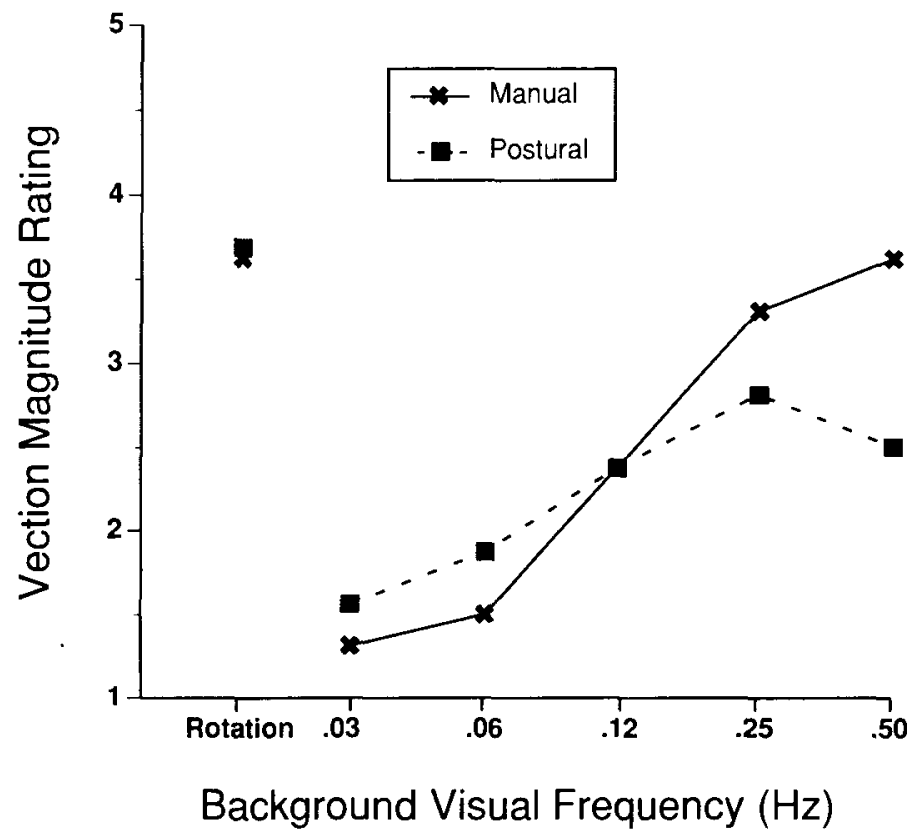

Figure 7. Vection magnitude ratings in the continuous rotation and five sinusoidal visual background conditions during the postural and manual tasks. Little or no vection was given a value of 1 , whereas 5 represented the highest amount of vection experienced. The data were averaged across all 8 subjects.

of the highly similar phase outputs of these two systems across the frequency spectrum. Also, the temporal tuning of roll vection was generally intermediate to the manual and postural tuning functions, although its peak depended on which task was being performed.

Our postural results largely replicated the findings of previous studies (Dichgans et al., 1976; Van Asten et al., 1988) that demonstrated a low-frequency dominance over visually elicited postural sway. Although the postural effect in our study peaked at $0.12 \mathrm{~Hz}$, good postural power was also exhibited at 0.03 and $0.06 \mathrm{~Hz}$. It should be noted that although Van Asten et al. did not measure postural changes below $0.10 \mathrm{~Hz}$, they predicted a falloff in postural power below this frequency because of the low visual velocities encountered in their constant-amplitude background motion. Dichgans et al. did observe lowpass postural effects, but their sinusoidal visual motion had constant peak velocities and varying amplitude (whereas we and Van Asten et al. used constant-amplitude but variable-velocity background motion). It has previously been shown (Clement et al., 1985) that postural sway in response to a continuously rotating background scene begins to diminish as the velocity falls below $20 \mathrm{dps}$ (the average velocity contained in our $0.25-\mathrm{Hz}$ motion).

It is somewhat more difficult to compare our manual results to those of Babler and Ebenholtz (1989). Using constant-amplitude sinusoidal roll motion of the background frame in a dynamic version of the rod-and-frame test, Babler and Ebenholtz showed very little gain change from 0.01 to $0.21 \mathrm{~Hz}$ when their data were averaged across all subjects. ${ }^{4}$ However, the nulling of perceived lod movement was the primary task in their study and was much more predictable than our compensatory tracking task, which required that the subject null an actual pseudorandom disturbance of the central display in the face of a secondary disturbance created by the sinusoidal movement of the visual background. Moreover, the visual impact of their oscillating frame was presumably far weaker than that of our large background visual field. It is noteworthy, however, that the subjects in Babler and Ebenholtz's study, who (like all of the subjects in the present study) experienced a substantial amount of vection, likewise showed a low-frequency rolloff in their gain functions. It is not clear, therefore, to what extent the experience of vection may have influenced the manually registered power spectrum in both our study and Babler and Ebenholtz's (see later discussion).

The postural and manual control systems also differed in their response to static tilt, which featured a sustained shift of the horizon and vertical scene gradient rather than movement of the background scene. Despite the stronger low-frequency response of the postural system, our tilted stimulus clearly biased the manual response to a greater extent. The magnitude of this bias was greater than is typically reported in the rod-and-frame test. Because the subjects were required to devote so little attention to the 
central display in the postural condition, the horizon shift may have been less effective because visual orientation mechanisms are generally believed to be more powerfully excited when the background scene is "ambient" (not directly attended). But the smaller postural tilt could also have been caused by the greater role of propriospinal and vestibulospinal reflexes in providing sustained postural support and a reference for the gravitational vertical in the standing position. Although a more powerful visual stimulus with an even larger FOV could have produced a significant static postural effect, such a demonstration would not negate the present finding that static tilt is a much more potent stimulus for the manual control system under the visual conditions used in our study.

Given the different masses and neural pathways of the postural and manual control systems, it is somewhat surprising that virtually no phase differences between them were obtained at any frequency in the present study. However, the phase data from this study do confirm previous postural (Dichgans et al., 1976; Van Asten et al., 1988; Xerri et al., 1988) and manual (Babler \& Ebenholtz, 1989) findings that the phases of both systems begin to seriously lag when the background visual modulation frequency rises above $0.10 \mathrm{~Hz}$. Longer phase lags with increasing temporal frequency would, of course, be expected of any system that contains a pure delay time.

What is somewhat unique about the present findings is that phase leads were observed in both responses at $0.03 \mathrm{~Hz}$ and, to a lesser extent, at $0.06 \mathrm{~Hz}$. It is not clear exactly why such leads occurred, as no phase leads were observed in the more predictable rod-and-frame task employed by Babler and Ebenholtz (1989). One possibility is that the postural and manual responses may have been able to overcome their initial lag at each phase reversal and eventually lead the background motion in this range (see Figure 2), because their velocities considerably exceeded that of the background motion at low frequencies. Another explanation for the phase leads is that the subjects may have responded primarily to the velocity of the visual background stimulus (which was advanced by $90^{\circ}$ relative to its position), given the velocity-dependence of visual orientation mechanisms (Clement et al., 1985). Finally, anticipatory phase leads cannot be ruled out in the case of the postural data, since the simple sinusoidal motion of the visual background could be predicted to some extent by the subject. But it is unlikely that the subjects could have readily predicted the motion of the visual background during the manual task, given the need to strenuously fixate the unpredictable central display motion. Thus, the manual phase lags could conceivably have been much smaller than the postural ones, if an equally complex (i.e., unpredictable) visual stimulus had been used in both conditions.

There are several possible explanations for the differences between the postural and manual power spectral tuning functions. One pertains to the smaller mass and more streamlined neural architecture of the manual system, which would seemingly favor a faster manual response whose power spectrum is shifted toward the medium-tohigh frequencies. While the phase lags of the two systems may not differ substantially, the visually mediated manual response to a velocity step increase is indeed "faster" in that it saturates within a few seconds (see Figure 2 of Weinstein et al., 1991), whereas the more sluggish postural response gradually builds up over a period of about 20-30 sec (see Figure 4 of Previc \& Mullen, 1991). A second explanation relates to the different baseline power spectra of the two systems in our study. That is, it is possible that the postural spectrum is naturally more lowpass than the broadband spectrum that we imposed on the manual control system by virtue of our forcing function. However, the visually driven manual response obtained in our study can hardly be described as "broadband," given its rather narrow peak at $0.25 \mathrm{~Hz}$. A third hypothesis for the postural-manual power spectral differences is more perceptually based. Previous research (Donnelly \& Previc, 1992) indicates that the manual control bias created by background visual roll motion is closely related to the perceived counterrotation of the central display that is induced by the background stimulus (see Duncker, 1929). Although display counterrotation is consciously perceived only when the central display is objectively stationary, the bias imposed by the subjects on the force-stick during the manual task may null a preconscious induced motion illusion. That is, the subjects realize that they are biasing the display in the direction of background motion but cannot explain why they are doing so or why it is so difficult for them to return the display to the desired horizontal position. The mediumfrequency peak of the manual control system is somewhat consistent with previous studies of the frequencydependence of induced motion. Nakayama and Tyler (1978), for example, showed that the lowest threshold for perceiving induced motion is between 0.5 and $2.0 \mathrm{~Hz}$ (with a sharp threshold increase below $0.5 \mathrm{~Hz}$ ), while Wallach and Becklen (1983) reported a steady decline in induced motion magnitude above $0.28 \mathrm{~Hz}$ (the lowest frequency tested in their study). The "perceptual" hypothesis described above is also consistent with evidence that manual control biases do not appear to utilize the vestibulospinal reflex pathways to the same extent as postural biases, but rather may be mediated largely by cortical mechanisms (Weinstein et al., 1991).

Finally, the difference between the postural and manual systems in their temporal response could have been an artifact of the differential positioning of the subject-the subject was seated rather than standing while performing the compensatory manual tracking task-and/or the fact that the central display in the manual task was both unstable and much larger. It is unlikely that the differential positioning of the subject was a major factor, since manual biases are unaffected by even larger positioning shifts, such as from upright to supine (Kenyon, unpublished data). The differential shape and motion of the central display was a more likely contributor to the postural-manual differences, for at least two principal reasons. First, the 
large tracking instability shown at the higher frequencies in the manual task created substantial central display motion, which could have diverted the subjects' attention away from the background scene and thereby enhanced its "ambient" effectiveness at those frequencies. Second, the central display motion could have increased the perceived velocity of the background scene by means of the same induced motion phenomenon as described above, and consequently could have augmented the scene's impact on the manual system. The possible role of differential display motion in creating the postural-manual differences will be addressed in Experiment 2.

One of the most interesting results of Experiment 1 was the fact that the frequency tuning of vection more closely resembled the manual tuning function than the postural one. This outcome does not necessarily challenge previously discussed findings that suggest a stronger link between visually induced postural sway and vection-both of which are "whole-body" responses-than between manual control and vection. But it is somewhat surprising that roll vection is more affected by higher frequencies than is the postural system, which has a much shorter onset latency to a visual velocity step increase (Previc \& Mullen, 1991). This paradox may be partly explained by the fact that vection elicitation is not required to perceive roll self-motion at low frequencies, because the otoliths veridically signal changes in the orientation of the head or body relative to gravity; whereas visually induced postural responses with their 1 -sec lag primarily serve to null out the low-frequency $(<0.10 \mathrm{~Hz})$ and low-amplitude $\left(<5^{\circ}\right)$ postural sway that occurs with the eyes closed (Dichgans et al., 1976; Van Asten et al., 1988; Xerri et al., 1988).

It should be noted that our vection results conflict with those of Lestienne et al. (1977), who found lowpass vection tuning with linear fore-aft stimulation, but not with those of Babler and Ebenholtz (1989), whose 3 vectionsensitive subjects also experienced their vection most intensely at a mid-to-high roll frequency $(0.21 \mathrm{~Hz})$. As noted earlier, however, the perception of self-motion at low temporal frequencies would not have to rely as much on visual mechanisms in the roll (and pitch) planes because the otolith organs adequately signal changes in the head and body relative to gravity, even at low frequencies. Thus, vection would be expected to be more dominated by low-frequency linear visual motion than low-frequency scene motion in roll or pitch.

An even more intriguing aspect of the vection results was the different temporal tuning obtained when vection was measured during the postural versus manual trials. This finding is by no means the first suggestion that the experience of vection can interact with other task parameters; as noted earlier, the gain and phase results in Babler and Ebenholtz's (1989) study differed, depending on whether or not their subjects experienced vection. There are several possible explanations for why visual frequency and task interacted for vection. One relates to the degree of task difficulty, which was greater at the low-to-mid frequencies in our postural task and at the mid-to-high frequencies in our manual one. Perhaps the greater task demands at certain frequencies reduced the amount of attention that the subjects devoted to the background scene. The second explanation involves the differential central display motion in the two tasks which, as noted above, may also relate to the amount of attention devoted to the background scene. A final explanation concerns the differential positioning of the subject, since there is some evidence that vection (unlike manual control) is altered when switching from an upright position to supine and other nonupright positions (Kenyon, unpublished data; Young, Shelhamer, \& Modestino, 1986; but see Watt \& Landolt, 1990). The role of differential display motion and subject positioning on the temporal tuning of vection will be addressed in Experiment 3 .

\section{EXPERIMENT 2}

The purpose of our second experiment was to determine if the postural-manual temporal tuning differences observed in Experiment 1 reflected true differences between the response properties of the two visuomotor systems, or were merely an artifact of the different central displays used in the two tasks. In this experiment, the subjects performed the postural task while viewing the central displays used in the previous postural and manual trials. When the "manual" control display was viewed, its motion was yoked to the corresponding motion recorded in the earlier manual trials.

\section{Method}

Subjects. Eight subjects participated in this experiment, including 5 who had served in the main experiment. The remaining subjects, who ranged in age from 23 to 37 years, replaced 3 original ones who were no longer available because of the long delay $(\sim 18$ months) between the main and follow-up experiments. The 3 new subjects fulfilled all of the visual and nonvisual criteria required of the original group. Their voluntary, fully informed consent was obtained, as required by Air Force Regulation 169-3

Procedure. The subjects performed the same postural task as in the main experiment, but were presented with only the five sinusoidal visual background conditions $(0.3,0.6,0.12,0.25$, and $0.50 \mathrm{~Hz})$. All background visual scenes were generated in the same manner as in the main experiment. The subjects viewed the scenes while fixating either the small red dot used in the previous postural trials or the moving central display used in the previous manual trials. For the 5 subjects who served in the main experiment, the central display retraced its movement during the first two manual trials of that experiment, on the basis of the recorded output from each trial. For the 3 replacement subjects, the central display motion was derived from manual tracking records obtained prior to the experiment at each of the five frequencies. In all other respects, the visual stimuli and postural recording methods in Experiment 2 were identical to those of Experiment 1 .

The postural data were collected over 2 days, with one complete replication (consisting of five frequencies $\times$ two display types) run per day. The five frequencies were randomly presented, with their 
presentation order reversed during the second set of trials. At each frequency, one display immediately followed the other in an alternating order.

\section{Results and Discussion}

The only postural measure that was quantified in Experiment 2 was the relative power of the postural response across the five temporal frequencies, which was used to compare the small dot versus moving display conditions. Unlike the power analysis in the main experiment, no baseline values were recorded that could be subtracted from the power values in each sinusoidal condition.

The results of this comparison are shown in Figure 8. It is clear that the substitution of the yoked display motion for the original fixation dot had no effect on the postural power spectrum. Interestingly, postural power in both conditions peaked at $0.03 \mathrm{~Hz}$, the lowest frequency tested, and fell off rapidly after $0.12 \mathrm{~Hz}$. (By comparison, the 3 new subjects' manual responses that were later fed back into the central display peaked at a much higher frequency $-0.12 \mathrm{~Hz}$-than did their postural responses.) The only significant effect revealed by Experiment 2's repeated measures ANOVA was visual frequency $[F(4,28)=30.21, p<.001]$. Because no frequency $\times$ display interaction effect was found, no individual $t$ tests were performed to assess differences between the two display means at any frequency.
The results of Experiment 2 conclusively demonstrate that the postural-manual temporal tuning differences found in Experiment 1 cannot be attributed to the different displays used in the two original tasks. Rather, the different temporal characteristics of the postural and manual systems are either due to their anatomical differences (i.e., different masses and neural architectures) or to their different functional roles (e.g., the postural system is designed to orient the body in space, whereas the manual system is designed to reach for and manipulate objects). As a consequence of these functional differences, any factor that might affect the perceived motion of an object-such as the inducing effect of a moving visual background-would necessitate a corresponding adjustment by the manual system, regardless of whether perceived whole-body motion has occurred or not.

It should be noted that postural power in the small fixation dot condition peaked at a lower frequency in Experiment 2 than in the main one, for reasons that are unclear. One explanation is that baseline postural power, which peaked at $0.03 \mathrm{~Hz}$, was subtracted only from the original data. However, overall baseline power at a given frequency was usually only a small fraction of the power recorded during sinusoidal stimulation and could not have greatly affected the relative power values. A second hypothesis is that the substitution of the 3 new subjects caused the shift, but this is also unlikely since the rela-

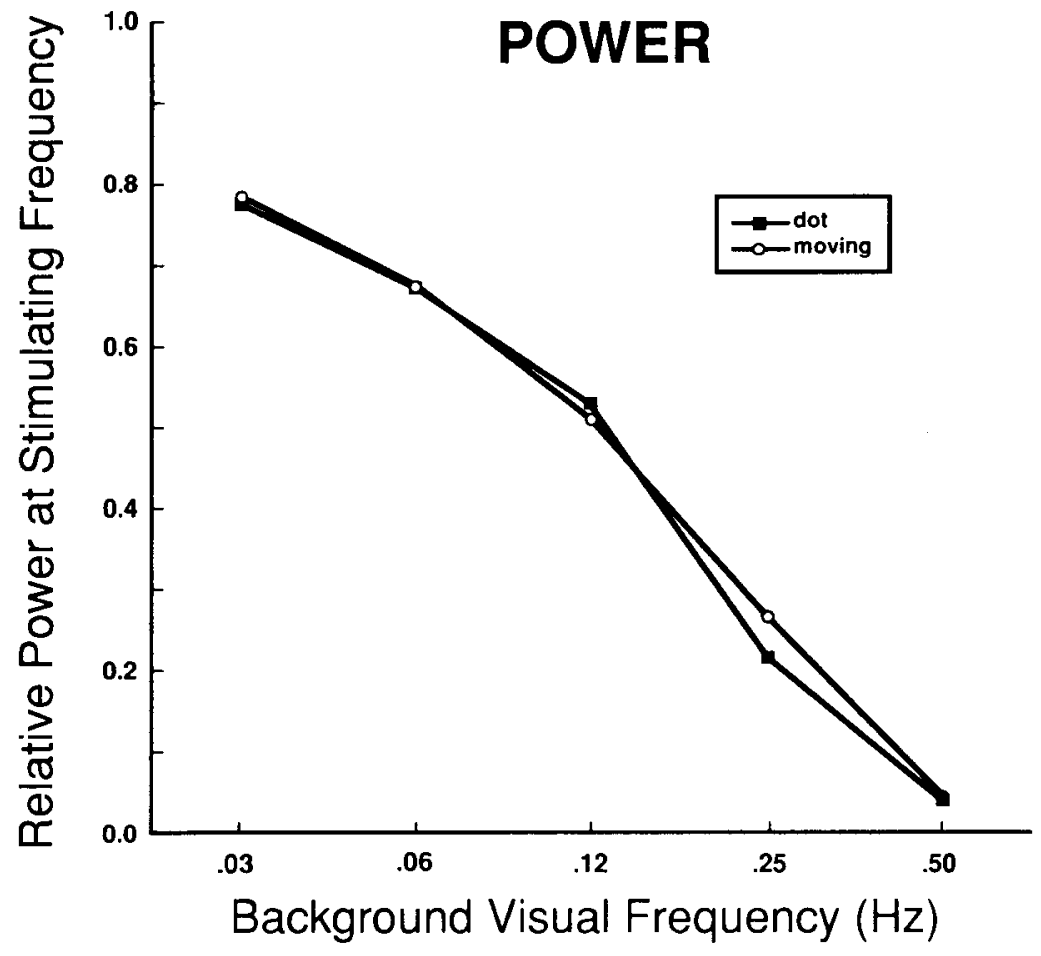

Figure 8. The mean relative power of the postural response in the five sinusoidal visual background conditions of Experiment 2 when a small dot (filled squares) or a moving display (open circles) was superimposed on the background visual scene. The mean relative power values were calculated for each of the 8 subjects and then averaged. 
tive postural power for the 5 original subjects also peaked at $0.03 \mathrm{~Hz}$ in Experiment 2.

\section{EXPERIMENT 3}

The purpose of our third experiment was to discover why vection ratings in the postural and manual trials of Experiment 1 differed in their temporal tuning. Two of the most likely sources for this discrepancy were the differential feedback provided by the two central displays and the differential positioning (standing vs. seated) of the subjects. Hence, vection measurements were recorded in Experiment 3 from seated and standing subjects who viewed either the small fixation dot or the larger central display whose motion retraced the original manual trial record.

\section{Method}

Subjects. The same 8 subjects who served in Experiment 2 participated in this experiment.

Procedure. The subjects were presented with the same visual background motion frequencies as in Experiment $2(0.03,0.06$, $0.12,0.25$, and $0.50 \mathrm{~Hz}$ ). They made their vection judgments after viewing each frequency for $15-30 \mathrm{sec}$, using the same scale that was employed in the main experiment. Unlike in Experiment 1, however, the subjects were not explicitly engaged in a postural or manual task when they made their vection judgments; rather, they experienced vection in a separate set of trials that followed each postural session of Experiment 2. The subject was seated in the same position as in the manual trials on half of the trials, and stood upright on the remaining half. In each position, half of the trials were run using the small fixation dot and the other half were run using the central display employed in the manual portion of Experiment 1.
The same "yoked" motion as that implemented in the moving display condition of Experiment 2 was used in these latter trials.

There were a total of 40 vection trials (five frequencies $\times$ two display types $x$ two positions $\times$ two replications). All the trials for a given position were performed in one session, while the other position was tested during the second session. One half of the subjects began in the seated position and the other half began in the standing position. Within a session, the visual background frequencies were randomly presented, and the presentation of display types was alternated for a given frequency. The same order of frequencies was used in the seated and standing positions, with the order reversed in each instance during the second replication.

\section{Results and Discussion}

The vection magnitude ratings recorded in the five sinusoidal conditions while the subject was either standing or seated and viewing either the small fixation dot or the yoked central display motion are shown in Figure 9. The differences among the four means were negligible below $0.50 \mathrm{~Hz}$, the frequency at which vection ratings overall proved marginally highest. But, at $0.50 \mathrm{~Hz}$ - the only frequency at which vection ratings in the postural and manual tasks differed in the main experiment-the moving central display produced elevated vection ratings in both the seated (3.19 vs. 2.81 ) and standing (3.94 vs. 3.25) positions.

The repeated measures ANOVA performed on the vection means contained three within-subjects factors: visual background frequency, display condition (dot vs. moving), and position (standing vs. seated). The ANOVA revealed a significant main effect of visual background frequency $[F(4,28)=25.89, p<.001]$ and a significant visual frequency $\times$ display interaction effect

\section{VECTION}

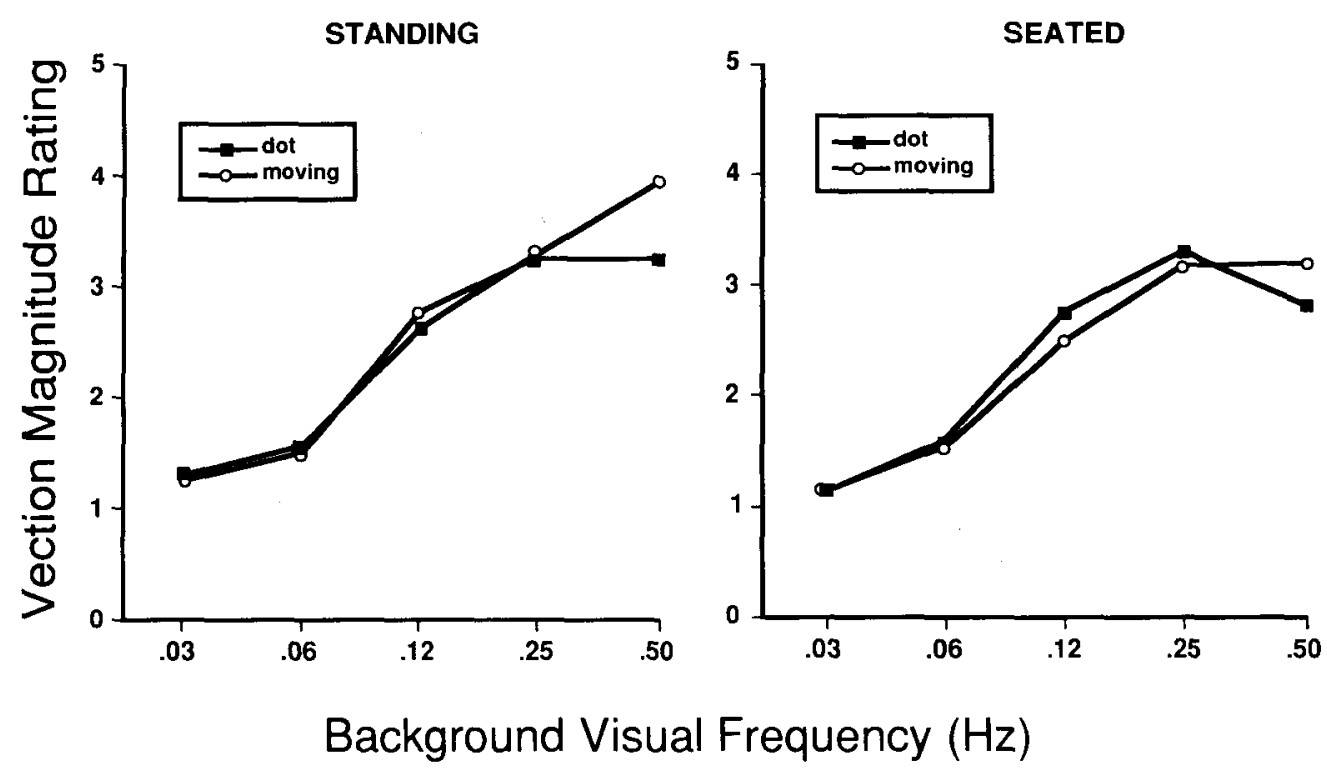

Figure 9. Vection magnitude ratings in the five sinusoidal visual background conditions of Experiment 3 when the subject was either standing (left panel) or seated (right panel) and viewing either a small dot (filled squares) or a moving central display (open circles). The data were averaged across all 8 subjects. 
$[F(4,28)=3.28, p<.05]$. No other main or interaction effects proved significant. Post hoc $t$ tests revealed that the display effect was significant only at $0.50 \mathrm{~Hz}$ $(p<.001)$.

The results of Experiment 3 indicate that the different vection ratings in the postural and manual tasks of Experiment 1 were largely caused by the different central displays used in the two tasks. More specifically, it appears that the movement of the central display was the factor most responsible for the task effect, given that vection ratings in Experiments 1 and 3 differed significantly only at $0.50 \mathrm{~Hz}$ (where manual control was most unstable and, consequently, the movement of the central display was greatest). As described earlier, it is possible that the substantial central display motion at the highest frequency captured our subjects' attention and moved it inward from the background visual scene, which thereby enhanced the scene's ambient effectiveness. Alternatively, the relative motion of the central display could, by means of the induced motion phenomenon, have increased the perceived velocity of the background display and thereby enhanced its ability to create an illusory perception of selfmotion. Such a mechanism would be expected to be most effective at $0.50 \mathrm{~Hz}$, where the manual tracking data exhibited a strong sinusoidal component at a nearly $180^{\circ}$ phase lag; hence, the induced counterrotation of the background display by the central one would have been in phase with the actual background motion at this frequency.

The failure of subject position to alter vection ratings demonstrates that the postural-manual vection differences at $0.50 \mathrm{~Hz}$ in the main experiment were probably not due to the seated position of the subjects while performing the manual task. Nor do task demands per se appear to have played a major role in the vection differences of Experiment 1 , since postural and manual vection ratings were comparable at low frequencies despite the greater postural difficulty (instability) present in this range. Nonspecific attentional demands on vection cannot be completely ruled out, however, given recent evidence that vection is a cortically mediated process (Heide, Koenig, \& Dichgans, 1990; Straube \& Brandt, 1987).

\section{GENERAL DISCUSSION}

The findings of this study demonstrate that the various visual orientation systems are far from equivalent in their temporal properties. Indeed, they differ from each other not only in their basic temporal tuning across frequencies, but also in response to several other temporal parameters. Our results also conflict somewhat with the classic view that the visual orientation system is uniformly dominated by low-frequency $(<0.10 \mathrm{~Hz})$ mechanisms, although the peaks of our visual effects would clearly fall well below those of their vestibularly generated counterparts.

Of the three systems examined in our study, manual control appears to exhibit the highest frequency tuning in response to sinusoidal visual roll stimulation. This outcome would not be predicted from the equivalent phase lags of the postural and manual systems, but it is consistent with the latter's much shorter saturation latency. The greater high-frequency response of the manual control system is also in line with its smaller mass and more rapid neural conduction. This is not to imply that the manual system cannot respond well at lower frequencies; indeed, a strong manual bias was created by the continuously rotating stimulus (whose average velocity was much greater than that of the low-frequency sinusoidal motions) and a weaker bias was created by the static tilted scene (which had virtually no effect on postural control). These findings suggest that a simple "bandwidth" algorithm cannot be invoked to explain all of the temporal tuning differences between the postural and manual systems that were found in Experiments 1 and 2.

The relationship between the temporal properties of the postural and vection systems is even more complex. Postural control and vection differ from manual control in that they (1) involve whole-body responses, (2) are mediated by the vestibulospinal reflexes, (3) have long saturation latencies, and (4) are more greatly affected by stimulation of the peripheral visual field. Yet, our vection tuning functions resembled the manual ones more closely than the postural ones, in that they typically peaked at or near the highest frequency tested. The higher frequency bandwidth of vection is even more surprising, given that the latency of vection onset to wide FOV visual motion is several seconds longer than the initial postural change (Previc \& Mullen, 1991). However, vection is presumably more dependent on cortically mediated perceptual mechanisms (Heide et al., 1990; Straube \& Brandt, 1987), as evidenced in our own study by the significant effect of the different central displays on vection (but not postural control) at $0.50 \mathrm{~Hz}$.

As alluded to earlier, a further complication in defining the temporal nature of visual orientation mechanisms involves the plane of motion, which was not manipulated in these experiments. The dynamic properties of visually mediated postural control, manual control, and selfmotion perception might be expected to differ on the basis of the varying contribution of nonvisual inputs in different motion planes. For example, visual information may be crucial to low-frequency orientational judgments in situations involving sustained circular motion about the gravitational axis or sustained linear motion (which are not adequately processed by either the semicircular canals or the otolith organs), but not in situations involving pitch or roll motion (which the otoliths continuously sense because these motions involve shifting of the body and/or head, relative to gravity). This could explain why our vection magnitude ratings were greatest in the mid-to-high frequency range, whereas Lestienne et al. 's (1977) linear vection ratings exhibited lowpass tuning.

The principal message of this study, then, is that considerable nonuniformity exists in the temporal properties of the various visual orientation systems. The major sources of this variability include the visuomotor system in question, the visual axis stimulated, and, to a lesser 
extent, the central visual displays used in testing the effects of the visual background scenes. Even for a particular orientational system, characterizations such as "sluggish" or "rapid" may be limited to the specific temporal parameter investigated (e.g., phase lag, saturation latency, or power spectral tuning). Finally, an examination of the specific dynamic properties of various visual orientation mechanisms may mask more fundamental relationships among them, such as the stronger overall link between vection and postural control (both of which involve wholebody mechanisms) than between vection and manual control (whose power spectral tuning functions to sinusoidal background visual roll stimulation are more similar). Thus, sweeping generalizations concerning the nature of human visual orientation systems are highly premature, pending careful investigation of these and other unresolved issues.

\section{REFERENCES}

Babler, T. G., \& Ebenholtz, S. M. (1989). Effects of peripheral circular contours on dynamic spatial orientation. Perception \& Psychophysics, 45, 307-314.

Berthoz, A., Pavard, B., \& Young, L. R. (1975). Perception of linear horizontal self-motion induced by peripheral vision (linearvection). Basic characteristics and visual-vestibular interaction. Experimental Brain Research, 23, 471-489.

BrandT, T., Wist, E. R., \& Dichgans, J. (1975). Foreground and background in dynamic spatial orientation. Perception \& Psychophysics, 17, 497-503.

Cheung, B. S. K., \& Howard, I. P. (1991). Optokinetic torsion: Dynamics and relation to circularvection. Vision Research, 31, 1327-1335.

Clement, G., Jacquin, T., \& Berthoz, A. (1985). Habituation of postural readjustments induced by motion of visual scenes. In M. Igarashi \& F.O. Black (Eds.), Vestibular and visual control on posture and locomotor equilibrium (pp. 99-104). Basel: Karger.

Delorme, A., \& MARTin, C. (1986). Roles of retinal periphery and depth periphery in linear vection and visual control of standing in humans. Canadian Journal of Psychology, 40, 176-187.

Dichgans, J., \& BRANDT, T. (1978). Visual-vestibular interaction: Effects on self-motion perception and postural control. In R. Held, $H$. W. Leibowitz, \& H.-L. Teuber (Eds.), Handbook of sensory physiology (Vol. 8, pp. 755-804). Berlin: Springer-Verlag.

Dichgans, J., Mauritz, K.-H., Allum, J.-H.-J., \& Brandt, T. (1976). Postural sway in normals and atactic patients: Analysis of the stabilizing and destabilizing effects of vision. Agressologie, 17C, 15-24.

Donnelly, M., \& Previc, F. H. (1992). Perceptual bias from fullfield motion follows induced motion, not vection. Investigative Ophthalmology \& Visual Science, 33, 1143.

DunCKer, K. (1929), Über induzierte Bewegung. Psychologische Forschung, 12, 180-259.

HeckmanN, T., \& Howard, I. P. (1991). Induced motion: Isolation and dissociation of egocentric and vection-entrained components. Perception, 20, 285-305.

Heide, W., Koenig, E., \& Dichgans, J. (1990). Optokinetic nystagmus, self-motion sensation and their aftereffects in patients with occipito-parietal lesions. Clinical Vision Sciences, 5, 145-156.

HowaRD, I. P. (1986). The vestibular system. In K. R. Boff, L. Kaufman, \& J. P. Thomas (Eds.), Handbook of perception and performance (Vol. 1, pp. 11-1-11-30). New York: Wiley.

HuANG, J.-K., \& YouNG, L. R. (1988). Visual field influence on manual roll and pitch stabilization. Aviation, Space, \& Environmental Medicine, 59, 611-619.

JeX, H. R., McDonnel, J. P., \& Phatak, A. V. (1966). A "critical" tracking task for manual control research. IEEE Transactions on Human Factors in Electronics, HFE-7, 138-145.
Kenyon, R. V., KNeller, E. W. (1993). The effects of field of view on the control of roll motion. IEEE Transactions on Systems, Man, \& Cybernetics, 23, 183-193.

Lestienne, F., Soechting, J., \& Berthoz, A. (1977). Postural readjustments induced by linear motion of visual scenes. Experimental Brain Research, 28, 363-384.

NaKayama, K., \& Tyle, C. W. (1978). Relative motion induced between stationary lines. Vision Research, 18, 1663-1668.

Ohmi, M., Howard, I. P., \& Landolt, J. P. (1987). Circular vection as a function of foreground-background relationships. Perception, 16, 17-22.

Previc, F. H. (1992). The effects of dynamic visual stimulation on perception and motor control. Joumal of Vestibular Research, 2, 285-295.

Previc, F. H., \& Mullen, T. J. (1991). A comparison of the latencies of visually induced postural change and self-motion perception. Journal of Vestibular Research, 1, 317-323.

RaPhan, T., \& Cohen, B. (1985). Velocity storage and the ocular response to multidimensional vestibular stimuli. In A. Berthoz \& G. Melvill Jones (Eds.), Adaptive mechanisms in gaze control (Vol. 1, pp. 123-143). Amsterdam: Elsevier.

ST. Cyr, G. J., \& Fender, D. H. (1969). Non-linearity of the human oculomotor system: Gain. Vision Research, 9, 1235-1246.

STRAUBE, A., \& BRANDT, T. (1987). Importance of the visual and vestibular cortex for self-motion perception in man (circularvection). $\mathrm{Hu}$ man Neurobiology, 6, 211-218.

Thoden, U., Dichgans, J., \& Savidis, T. (1977). Direction-specific optokinetic modulation of monosynaptic hind limb reflexes in cats. Experimental Brain Research, 30, 155-160.

Van Asten, W. N. J. C., Gielen, C. C. A. M., \& Denier VAN DER GoN, J. (1988). Postural movements induced by rotations of visual scenes. Journal of the Optical Society of America A, 5, $1781-1789$.

Vos, J. J. (1960). Some new aspects of color stereoscopy. Journal of the Optical Society of America, 50, 785-790.

WALLACH, H., BECKLEN, R. (1983). An effect of speed on induced motion. Perception \& Psychophysics, 34, 237-242.

WatT, D. G. D., \& LANDOLT, J. P. (1990). Effects of short-term weightlessness on roll circularvection. In Situational awareness in aerospace operations (pp. 20-1-20-6). Neuilly sur Seine, France: AGARD.

Weinstein, L. F., Previc, F. H., Simpson, C. G., Lyons, T. J., \& Gillingham, K. K. (1991). A test of thumb and index finger control in overcoming a visual analogue of the giant hand illusion. Aviation, Space, \& Environmental Medicine, 62, 336-341.

Xerri, C., Borel, L., BARTHElemy, J., \& LACOUR, M. (1988). Synergistic interactions and functional working range of the visual and vestibular systems in postural control: Neuronal correlates. Progress in Brain Research, 76, 193-203.

Young, L. R., Shelhamer, M., Modestino, S. (1986). M.I.T./ Canadian vestibular experiments on the Spacelab-1 mission: 2 . Visual vestibular tilt interaction in weightlessness. Experimental Brain Research, 64, 299-307.

ZacharIAS, G. L., \& Young, L. R. (1981). Influence of combined visual and vestibular cues on human perception and control of horizontal rotation. Experimental Brain Research, 41, 159-171.

\section{NOTES}

1. A major difficulty in attempting to equate the visual stimuli used in the postural and manual tasks is that visually induced postural shifts rarely exceed a few degrees (Clement et al., 1985; Van Asten et al., 1988), whereas the effect of a background visual scene on manual control can easily exceed the angular displacement of the stimulus itself ( $20^{\circ}$ in this instance). Hence, yoking the visual background motion to the motion of the stick could be realistically achieved only in the baseline and tilt conditions. Had visual background feedback congruent with the movement of the central display been superimposed on the continuous rotation and sinusoidal motion in the manual trials, the manual control task would have become so unstable that the subjects could not have completed it.

Another potential difficulty in comparing the postural and manual systems in roll relates to the fact that visual roll stimulation results in both 
a linear and angular postural adjustment, because the postural center of rotation usually resides at the ankles whereas the visual scene rotates about the visual axis. Physiologically, however, rotation about the visual axis produces a highly similar vestibulospinal response to that generated by a natural fall in the lateral (roll) plane (Thoden et al., 1977).

2. The external disturbance signal was designed to simulate white noise and thereby evoke a wide-frequency manual output from the subject. Without it, the subject would have responded only to frequencies near the natural peak of the closed-loop system. The external forcing function was created by summing 13 sinusoids, each of which was a consecutive prime harmonic of $0.007 \mathrm{~Hz}$ (beginning with the seventh harmonic). The power spectral density of the total disturbance signal was approximated by a first-order function with a break frequency of $3.14 \mathrm{~Hz}$. The phases of the sinusoids were set randomly, and the net value of the cumulative forcing disturbance equaled zero by the end of each trial.

3. The Fourier transform (FT) at each of the stimulating frequencies was calculated using the formula:

$$
\operatorname{FT}(D, k)=\frac{1}{\sqrt{N}} \sum_{n=1}^{N} D(n) \times e^{-j w / k) n h},
$$

where $D$ is the sampled signal, $k$ is the index to the specific frequencies in $W, n$ is the time step, $h$ is the time interval, and $N$ is the number of samples of $D$. The power at the specific frequencies in the $D$ is calculated by the following formula: $\operatorname{Power}(D, k)=\mathrm{FT}(D, k) \times \mathrm{FT}(D, k)^{*}$, where ${ }^{*}$ denotes the complex conjugate response.

In contrast to the very slight movement of the visual background in the postural baseline condition, the visual background in the manual baseline condition moved much more substantially because it was congruent with the inherently more unstable central display (made so because of the lambda and forcing functions). But even though the motion of the visual background in the two baseline conditions could not be made equivalent, the baseline power at the stimulating frequency typically represented less than $5 \%$ of the power derived from the sinusoidal visual background motion itself. Thus, it may be concluded that any differences between the baseline conditions probably had little impact on the power analysis.

4. Six of Babler and Ebenholtz's subjects (the nonvection-sensitive ones) showed a high-frequency rolloff above $0.013 \mathrm{~Hz}$ (the lowest frequency tested), whereas 3 subjects (the vection-sensitive ones) showed a low-frequency falloff below $0.213 \mathrm{~Hz}$ (the highest frequency tested).

(Manuscript received July 25, 1991;

revision accepted for publication November 20, 1992.) 